\title{
An Exploratory Comparison of the Roles of Representations in Face to Face and Online Collaborative Learning
}

\author{
Daniel D. Suthers, Christopher D. Hundhausen, and Laura E. Girardeau \\ Laboratory for Interactive Learning Technologies \\ Department of Information and Computer Sciences \\ University of Hawai $i$ at Manoa \\ \{suthers,hundhaus,girardea\}@hawaii.edu
}

\begin{abstract}
The paper reports an empirical study comparing the role of discourse and knowledge representations in face to face versus distance collaborative learning. The study reported in this paper compares Proximal (face to face) with Distal (synchronous collaboration via networked software) conditions. Two competing hypotheses were evaluated: (1) The influence of representations in the Distal condition could be weaker because of the lack of implicit "taken as shared" that results from working together in front of a physically shared display, and because of the greater difficulty of utilizing the representations as a resource for conversation through gesturing. (2) The influence of representations in the Distal study could be stronger because participants may rely more on them for their communication in the absence of face-to-face communication. The quantitative data supported the second hypothesis, while other observations supported the first hypothesis. A synthesis is offered.
\end{abstract}

\section{Introduction}

Online collaborative learning intrinsically requires that learning be mediated by external representations. These representations may include discourse representations (e.g., the chat rooms and threaded discussion tools by which learners and teachers communicate in their native language [5], disciplinary representations such as visualizations and designed artifacts (e.g., [7, 11]), and symbolic representations of one's theories and reasoning that we will term knowledge representations (e.g., [16]). Unlike the spoken discourse of proximal collaboration, the discourse in distance collaboration takes place in a software-supported representational medium. Given the total reliance on external representations in online collaborative learning, it is appropriate to ask how these representations should be designed to facilitate collaboration.
Our prior work on external representations in face-toface collaborative learning situations has shown that differences between representational notations can translate into differences in the focus of learners' discourse and collaborative activities $[14,15]$. In these studies, learners were asked to solve a problem in science or public health, presented as a sequence of information pages, while utilizing one of several experimentally manipulated alternate representations (text, graph, and matrix) for recording data, hypotheses, and the evidential relations between them. Differences in discourse focus were predicted according to the kinds of information that the representations prompt one to seek, and the information needs that become salient as one constructs a representation [12]. We found that visually structured and constrained representations can provide guidance for collaborative learning that is not afforded by plain text. However, more guidance is not necessarily better. For example, the matrix representation prompted for consideration of irrelevant relationships as well as relevant ones. The graph representation - "evidence maps" - provided guidance without excessive prompting. Benefits of graphical evidence maps have also been observed in a classroom setting [17].

This paper reports our first extension of this work to a study of how representations might influence collaboration in distance collaboration situations. We begin with a comparison of face-to-face and online collaboration, since we have studied the former in great detail and need to understand how online collaboration differs. This is not a new topic. Extensive prior research has compared performance of face-to-face collaborators to performance of users of various forms of technologymediated communication (including audio, video, and various forms of computer mediated communication). Studies of synchronous problem solving generally show degradation of both problem solving performance and interpersonal communication due to the reduced "bandwidth" or available modes of interaction associated with technology-mediated communication [4.9], although 
factors extrinsic to the technology itself may play a role [19], However, it would be overly simplistic to treat technology mediation as an atomic variable, or to evaluate this variable apart from task characteristics or objectives [2]. Indeed, an extensive literature on asynchronous online learning typically concludes that there is no significant difference in learning outcomes as compared to traditional classroom learning [10].

It was not our intent to replicate or test these results. Rather, we sought to understand how the roles that representations play might change between face-to-face and online collaboration in order to understand how our previous work might inform the design of online learning environments. Our previous study provided a baseline understanding of how a representation such as Graph is appropriated and used by learners and influences learner's activities in a face-to-face setting. Would the role of Graph be similar when interaction is online, with a textual "chat" tool replacing spoken language?

The conditions compared in this study are termed Proximal (face to face) and Distal (synchronous collaboration via networked software) in analogy to the medical terms. We approached this work with two hypotheses in mind. At the outset of the study we considered these hypotheses to be competitors with each other, but had no prediction concerning which would be the dominant factor.

(H1) The "Co-present Affordances" Hypothesis: The influence of representations in the Distal condition will be weaker because some key representational affordances for collaboration depend on working together in front of a physically shared display, including implicit "taken as shared" context and use of the representations as a resource for conversation through gestural deixis (referring to something in the extralinguistic context [3]).

(H2) The "Appropriation for Communication" Hypothesis: The influence of representations in the Distal study will be stronger because participants must place greater reliance on those representations for their communication to compensate for the absence of face-to-face communication.

The results show that the distribution of activity was quite different between the Proximal and Distal groups. Quantitative analysis of this distribution largely supports the second hypothesis. However, other observations indicated that influences predicted by the first hypothesis are also manifest in different aspects of participants' activity. We discuss alternate explanations for these results, and broader implications for the nature of collaborative learning with knowledge representations.

\section{Design}

The present study built on a prior study that compared three representations: matrix, graph, and text $[14,15]$. We elected to implement a synchronous distance collaboration version of the graph condition and to compare performance of participants in this condition with those in the previous proximal (face to face) graph condition. Both groups were given the identical task of exploring an unsolved "challenge problem," presented as a series of textual web pages, by recording data, hypotheses, and evidential relations as they encountered them.

\subsection{Participants}

Twenty (20) students were recruited in self-selected, same-gender pairs, out of introductory biology, chemistry, physics, and computer science courses at the University of Hawai' i. Participants were age 25 years or younger and native English speakers. Participants were paid a \$25 honorarium. Pairs of participants were randomly assigned to treatment groups.

The unit of analysis was the pair (all individual scores were aggregated for each pair). We used pairs rather than larger groups because a pilot study showed that participation of students is reduced when there are more than two students at a single keyboard and monitor in the Proximal condition. We used same-gender pairs because we observed censoring problems with mixed-gender pairs in our pilot study. We used self-selected pairs (friends) to avoid the additional social familiarization that would have been required between strangers.

\subsection{Materials}

Pairs of participants used one of two different versions of software for representing data, hypotheses, and evidential relations. The Distal version is shown in Figure 1. Participants used the upper right hand window to move forward through a sequence of 15 pages that presented information relating to the cause of a mysterious neurological disease on the island of Guam. The left-hand window contained a graphical tool for constructing representations of the data, hypotheses, and evidential relations participants gleaned from the information pages on the right. The graph tool is based on Belvedere [16], and enables one to build a graph of nodes expressing data items and hypotheses, and links labeled "+," “-," or "?" representing evidential relations. 


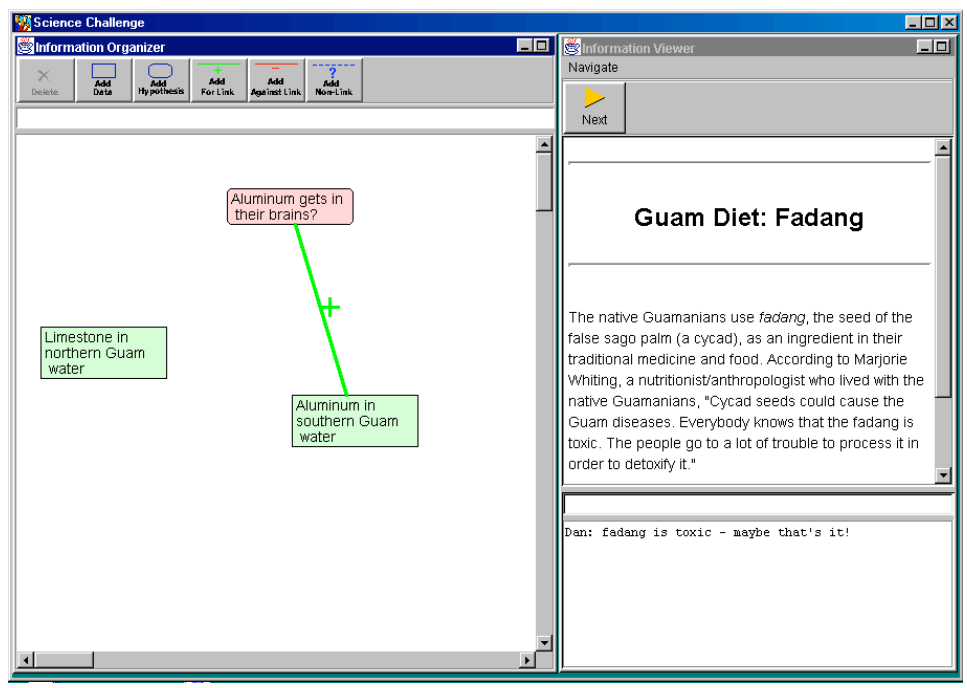

Figure 1. Experimental software with chat tool

The software for the Distal condition provided a simple chat tool in a window in the lower right (which was not present in the Proximal version). Messages typed into a chat entry box were sent to both participants' shared chat displays once the message was completed and the "send" button was pressed.

Both versions of the software support deixis by causing the color of objects to change when one passes the cursor over them, enhancing the deictic value of the cursor. The Distal version of the software replicated these color changes to the remote display. In this manner we attempted to support the use of the knowledge representation as a resource during conversation in the Distal as well as Proximal condition.

\subsection{Procedure}

At the beginning of the learning session, participants (co-present in both conditions) were given a brief (10minute) introduction to the software they would be using. At the conclusion of this introduction, one of the Distal participants was led to a different room containing an identical computer setup. Then, in both conditions, participants worked on a warm-up problem (on mass extinctions) that was unrelated to the main problem, so that they could become acquainted with the software and the information-recording process. After 12 minutes, participants were instructed to stop work on the warm-up problem, and to move on to the main problem (on the neurological disease). Participants were given as much time as they needed to explore all 15 pages on the main problem. When they reached the page that informed them that there were no more pages left, the experimenter asked them whether they felt they were done. Some participant pairs decided that they wanted to work further on their representations; they were given as much additional time as they needed. Once a participant pair declared themselves done, the experimenter instructed them to turn off their computer screen(s), at which point they were given 20 minutes to individually complete a multiplechoice post-test. (We did not have any specific predictions about the outcome of this test, but administered it to keep the conditions the same as Proximal.) At the conclusion of this test, the Distal participants were brought back together. In both conditions, participants then were allotted 30 minutes to complete a collaborative essay using a word processor.

\section{Results}

Posttests of memory for factual information showed no significant difference between Proximal and Distal groups. We are currently analyzing the essays. In this paper we focus on a categorical analysis of the verbal and representational acts of the participants.

\subsection{Data Analysis}

Video/audiotape of the proximal sessions were transcribed by hand. The software generated transcripts of the distal sessions automatically. Transcripts were divided into "segments," each consisting of a verbal or typed utterance (multi-propositional utterances were divided into individual segments) or a change to the representation. See [14] for details of the coding used. Then we performed a content analysis of participants' learning processes by coding all segments in the 20 transcripts into the following mutually exclusive "topic" categories: 
- Evidential relation. These segments consider whether data and hypotheses are consistent, that is, whether a data item supports or conflicts with a hypothesis. For example, the segment "That's for the genetics hypothesis" would be coded as evidential relation-consistency.

- Epistemic classification. These segments classify information as either empirical or theoretical - that is, as either data or hypothesis. For example, the segment "Let's make a hypothesis about toxic drinking water" would be coded as epistemic classification. Likewise, in the graph software, the action of clicking on the "create data" button would be coded as epistemic classification.

- Hypothesis statement. This coding was applied when participants stated a hypothesis concerning a possible explanation for the disease without labeling it as a hypothesis (e.g., "Might be a combination of both").

- Metacognitive. In these segments, participants step back and either assess what they know so far (e.g., "We know that they used the drinking water for the fadang, to prepare the seeds"), or identify information that is needed but lacking (e.g., "See, but it doesn't say that these admission records are patients that have the disease").

- Warrant. These segments provide justification for an evidential relation previously cited. For example the second half of, "That supports the aluminum hypothesis, because Irian Jaya was found to have higher than normal levels of aluminum in the soil" would be classified as a warrant.

- Tool talk. These segments discuss some aspect of the software. Participants might, for example, ask how to complete some specific task with the software (e.g., "How do you get this out of the way?"); they might complain about the software (e.g., "Oh my, what's wrong with this thing?"), or they might share their emerging understandings of how the software works (e.g., "If we click on this we can see it").

- Domain talk. These segments discuss the domain of the science problem that participants are exploring. This category had the lowest precedence: we coded segments into this category only if they could not be coded into one of the five categories above. For example, "Northern Guam is a low limestone plateau" would be coded as domain talk.

- On-task. These segments did not fall into any of the first six categories, but could still be considered ontask. For example, "Let's go to the next page" would be coded as on-task.
- Off-task. These segments were deemed to be unrelated to participants' learning task. For example, "What did you do last night?" would be considered off-task.

In addition, we coded topic segments with "modifier" categories, according to whether they were

- Verbal or representational-spoken or expressed in the chat tool, versus represented using the software;

- Recited or non-recited - quoted verbatim from the information pages, or not quoted;

- Introduced or repeated - the first occurrence of an idea within a given conversation, or a reintroduction of an idea already brought up within a given conversation.

In order to verify the reliability of our coding system, we had two independent analysts code $20 \%$ of the transcripts. With respect to the eight mutually exclusive "topic" categories, our analysts attained $89 \%$ overall agreement, and 0.86 kappa. With respect to the modifier categories, agreement levels ranged from $88 \%$ (0.77 kappa) for introduced vs. repeated, to $100 \%$ agreement (0.99 kappa) for verbal vs. representational. We decided that our coding system was sufficiently reliable, and had a single analyst code the remaining $80 \%$ of the transcripts.

\subsection{Quantitative Results}

The distribution of the codes across treatment groups is shown in Table 1. There were many more segments in Proximal than in Distal, this difference being attributable to larger counts in most categories, particularly in domain talk and other on task talk, although Distal participants engaged in more epistemic classification and hypothesis statement acts.

Table 1. Distribution of codes by raw count

\begin{tabular}{|l|r|r|}
\hline & Proximal & \multicolumn{1}{l|}{ Distal } \\
\hline Evidential Relation & 569 & 561 \\
\hline Epistemic Classification & 439 & 644 \\
\hline Metacognitive & 37 & 28 \\
\hline Warrant & 31 & 5 \\
\hline Tool Talk & 268 & 85 \\
\hline Domain Talk & 1618 & 368 \\
\hline Hypothesis Statement & 38 & 76 \\
\hline On Task & 1639 & 987 \\
\hline Off Task & 159 & 144 \\
\hline Totals & 4798 & 2898 \\
\hline
\end{tabular}




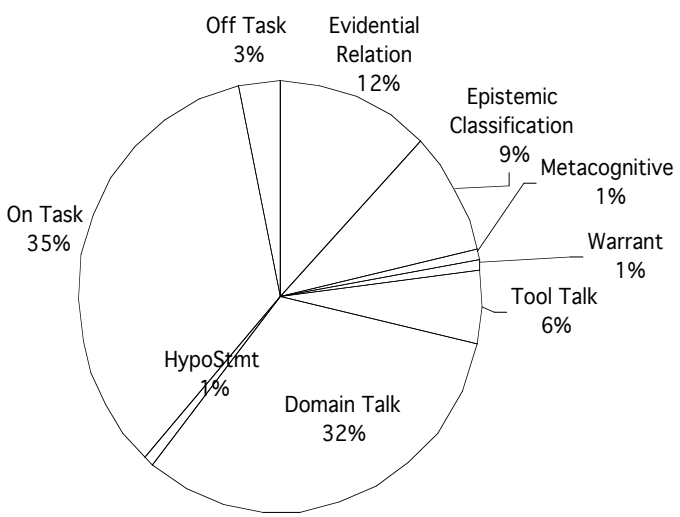

Proximal

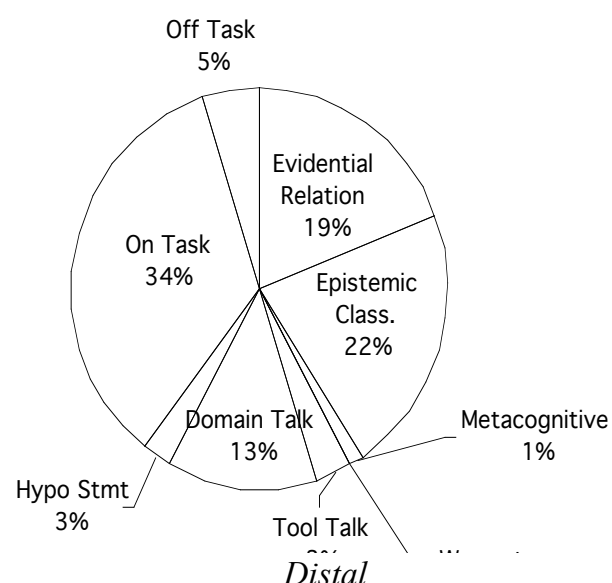

Distal

Figure 2. Percentages of each category compared

Table 2. Breakdown of on-task, non-Recited segments

\begin{tabular}{|c|c|c|c|c|c|c|}
\hline \multirow{2}{*}{\multicolumn{2}{|c|}{ Code }} & \multicolumn{2}{|c|}{ Total Counts } & \multicolumn{3}{|c|}{ Mean Counts per Pair and p-levels } \\
\hline & & Proximal & Distal & Proximal & Distal & $\mathbf{p}$ \\
\hline On-Task, Non-Re & ted & 4530 & 2719 & 453.0 & 271.9 & 0.0613 \\
\hline & Verbal & 3646 & 1556 & 364.6 & 155.6 & 0.0126 \\
\hline & Representational & 884 & 1165 & 88.4 & 116.5 & 0.2232 \\
\hline
\end{tabular}

Table 3. Breakdowns of evidential and epistemological classification acts

\begin{tabular}{|c|c|c|c|c|c|c|}
\hline \multirow{2}{*}{\multicolumn{2}{|c|}{ Code }} & \multicolumn{2}{|c|}{ Total Counts } & \multicolumn{3}{|c|}{$\begin{array}{l}\text { Percentage of On-Task, Non-Recited, } \\
\text { and p levels }\end{array}$} \\
\hline & & Proximal & Distal & Proximal & Distal & p \\
\hline \multicolumn{2}{|c|}{ Evidential Relations } & 569 & 561 & 12.56 & 20.15 & .0155 \\
\hline & Verbal & 314 & 35 & 6.93 & 1.28 & .0001 \\
\hline & Representational & 255 & 514 & 5.63 & 18.87 & .0002 \\
\hline \multicolumn{2}{|c|}{ Epistemic Classification } & 439 & 644 & 9.69 & 22.83 & .0007 \\
\hline & Verbal & 120 & 24 & 2.65 & 0.88 & .0050 \\
\hline & Representational & 319 & 598 & 7.04 & 21.95 & .0004 \\
\hline \multicolumn{2}{|l|}{ Domain Talk } & 1618 & 368 & 35.72 & 13.53 & .0002 \\
\hline & Verbal & 1311 & 367 & 28.94 & 13.50 & .0005 \\
\hline & Representational & 307 & 1 & 6.78 & 0.04 & .0001 \\
\hline \multicolumn{2}{|c|}{ Hypothesis Statement } & 38 & 76 & 0.84 & 2.80 & .0409 \\
\hline & Verbal & 38 & 75 & 0.84 & 2.76 & .0409 \\
\hline & Representational & 0 & 1 & 0.00 & 0.04 & .3173 \\
\hline
\end{tabular}

The percentages of each category relative to the total count for each group are shown in Figure 2. We performed statistical tests on the percentages rather than the raw counts to control for differences in verbosity between groups, although raw counts will be consulted in our interpretation of the results (next section). The most striking differences in the percentages are in domain talk, epistemic classification and (to a lesser degree) evidential relation. The Proximal participants engaged in a greater percentage of domain talk, which is reflected in the raw 
Proceedings of the 36th Hawai'i International Conference on the System Sciences (HICSS-36), January 6-9, 2001, Hilton Waikoloa Village, Hawai'i (CD-ROM), Institute of Electrical and Electronics Engineers, Inc. (IEEE).

counts as well. Distal participants engaged in a greater percentage of epistemic classification, evidential relation and hypothesis statements. The raw counts for evidential relation are actually very similar.

For the purposes of this study, "verbal" means spoken in the Proximal condition and use of the chat tool in the Distal condition; while "representational" means use of the graph tool in both conditions. Table 2 compares the two conditions with respect to their counts of On-Task, Non-Recited segments. An independent samples t-test indicates that the Proximal condition had significantly more verbal segments than the Distal condition $(\mathrm{df}=1, \mathrm{~T}$ $=-2.77, \mathrm{p}=0.0126)$; no other statistically significant differences were detected.

Table 3 shows both raw counts and percentages of the On-Task, Non-Recited segments listed in Table 2. (Therefore the percentages differ slightly from those in Figure 2, which are of the total number of segments).

To test for statistically significant differences, we compared each Proximal/Distal pair of percentages using a Kruskal-Wallis ANOVA. As indicated in Table 2, differences between all of the values shown except for the last row are statistically significant at levels ranging from $\mathrm{p}=.0001$ to .05 . The overall pattern is as follows. The Proximal participants engaged in a greater number of ontask, non-recited acts overall, with most of these being spoken. Distal participants engaged in a greater percentage (although approximately the same raw count) of acts concerned with relations of evidence. These acts occurred more in the verbal (spoken) medium in Proximal, and in the representational (graph) medium in Distal. Similarly, Distal participants engaged in a greater percentage of epistemological classification acts, these being primarily in the representational medium (as opposed to the chat tool). However, Proximal participants engaged in considerably more talk about domain attributes and relationships.

\subsection{Qualitative Observations}

Examination of the transcripts during analysis led to the following qualitative observations concerning the timing and purpose of discourse related to the representations. We offer the following impressions and examples to provide the reader with a feel for the interactions, while recognizing that formal analysis and testing would be needed to substantiate our impressions.

For Proximal participants, the graph functioned as a stimulus to discourse, and the representations were also treated as an external product of the discourse. Agreement tended to be forged before representations were made, as in the following example leading up to the events at time 10:42. The graph also served well as a deictic resource. Coordination between discourse mode and diagrammatic media was easier for Proximal participants because they could simply point to an item on the screen or click their cursor when determining a relationship or discussing an issue:

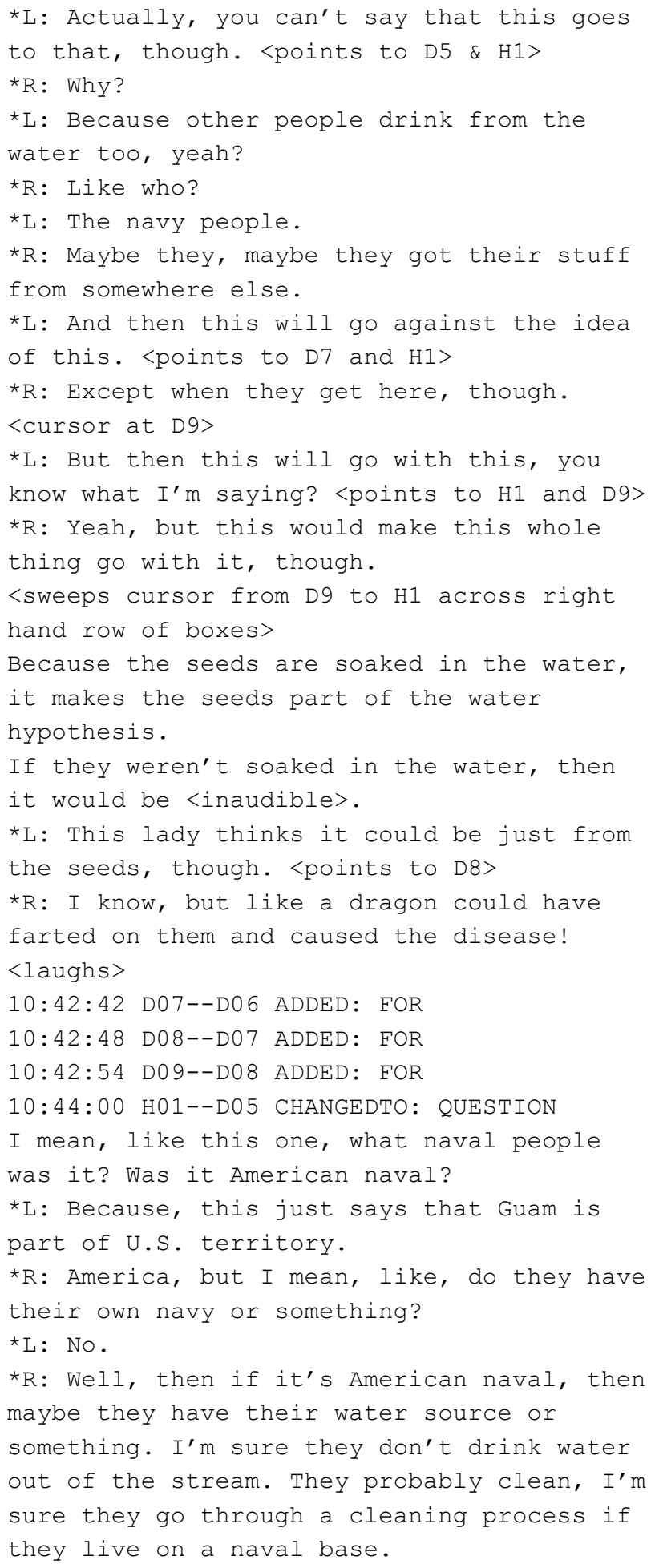


Proceedings of the 36th Hawai'i International Conference on the System Sciences (HICSS-36), January 6-9, 2001, Hilton Waikoloa Village, Hawai'i (CD-ROM), Institute of Electrical and Electronics Engineers, Inc. (IEEE).

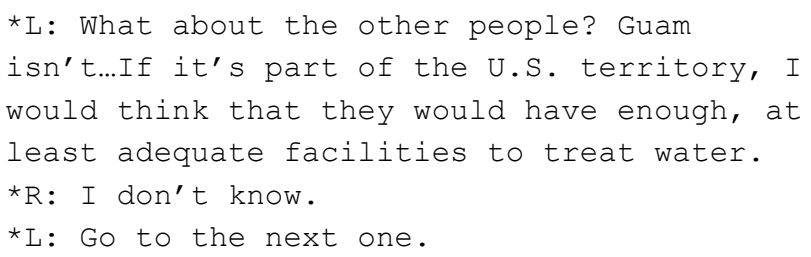

For Distal participants, the graph also functioned as a stimulus to and product of discourse, but was also used to a much greater extent as a discourse medium. Discourse often took place within the graphs in two ways: (1) participants proposed new items or relations by creating them in the graph medium, whereupon chat focused on approval or disapproval; and (2) participants used the graphical representation in place of the chat tool to send a message that was deleted. For instance, one pair posted on-task questions, such as "What should we do now?" and "I don't know what the answer is" in the graph rather than in chat. Another pair posted domain questions such as, "What is causing this disease?" and linked them up with data and hypothesis shapes. At times the graph was used in a manner peripheral to discourse, when a participant independently modified the graph amidst unrelated chat discussion (the graph was rarely modified without related talk in Proximal). This created a need for the removal of duplicate or similar items created independently by each partner, and negotiation of which item would be removed.

The following example, although far more verbose than most Distal exchanges, serves well to illustrate these typical characteristics of Distal discourse. Instead of discussing what to add to their graph in advance, the pair proposes new items or relations by first representing them independently of one another in the graph medium. Chat then focuses on short comments of approval or disapproval and negotiation about duplicates:

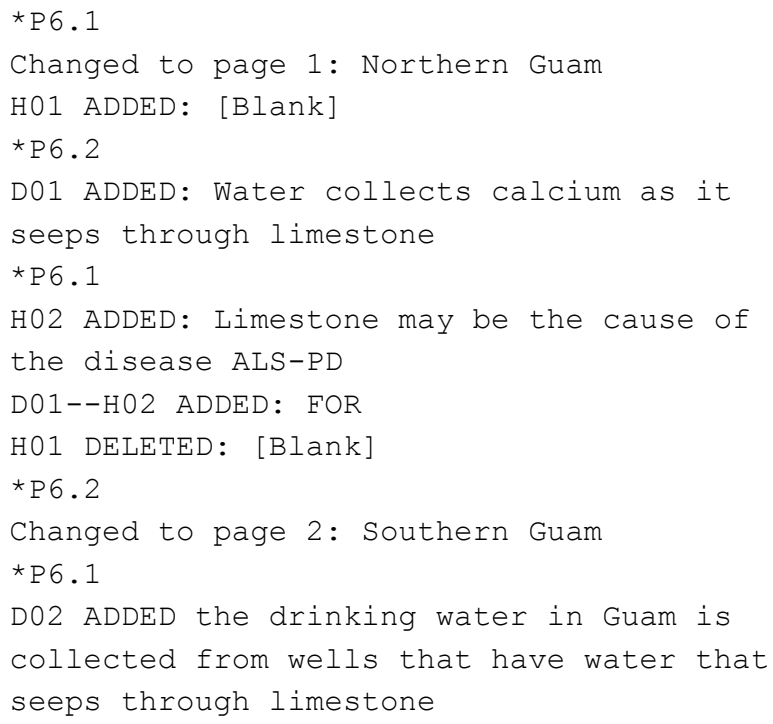

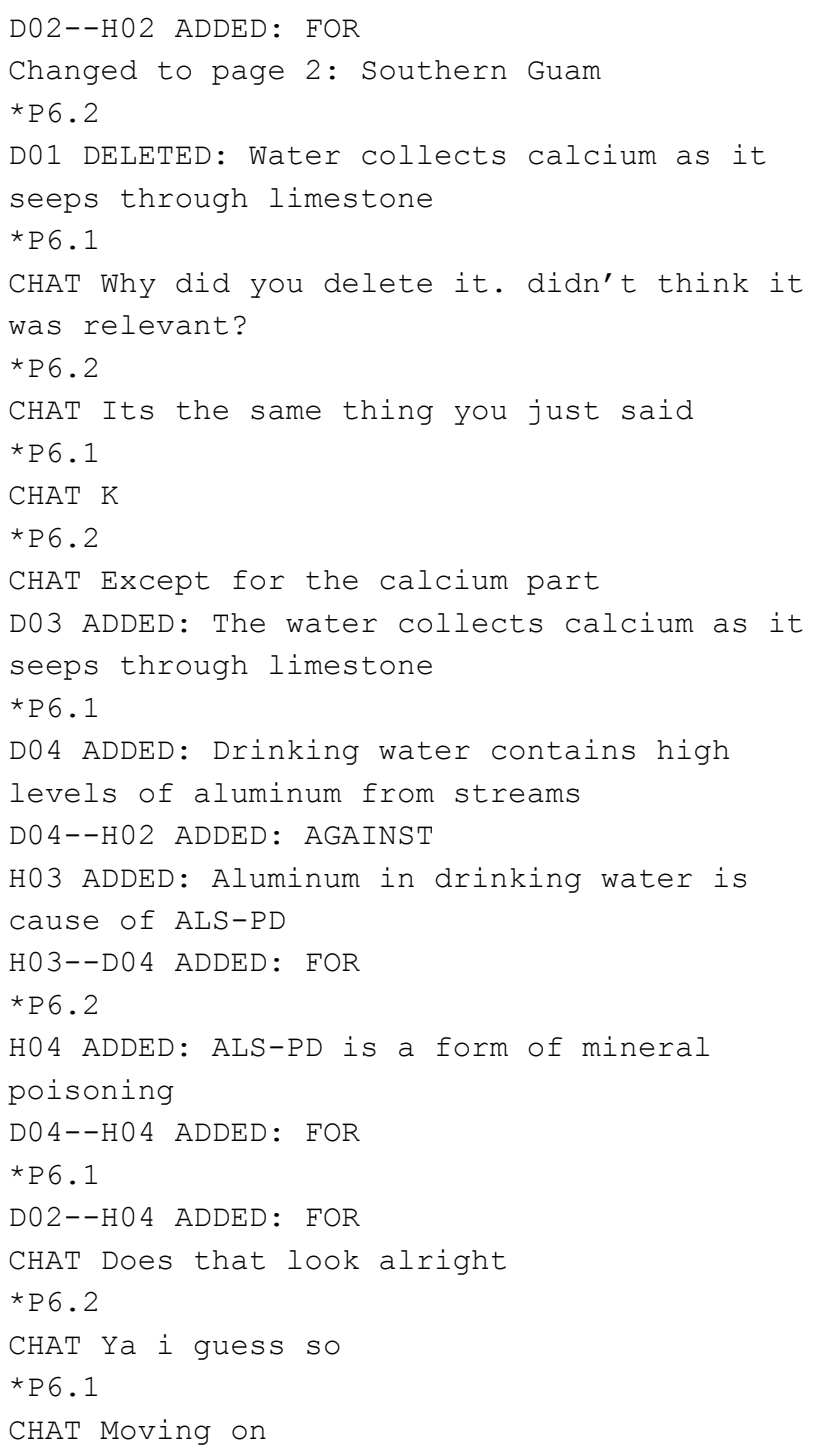

Overt discussion of alternate hypotheses in Distal was often concentrated at the end of the session, after all representations had been made and participants were urged to come to a final conclusion. This may explain the higher counts for statements of hypothesis in the Distal condition.

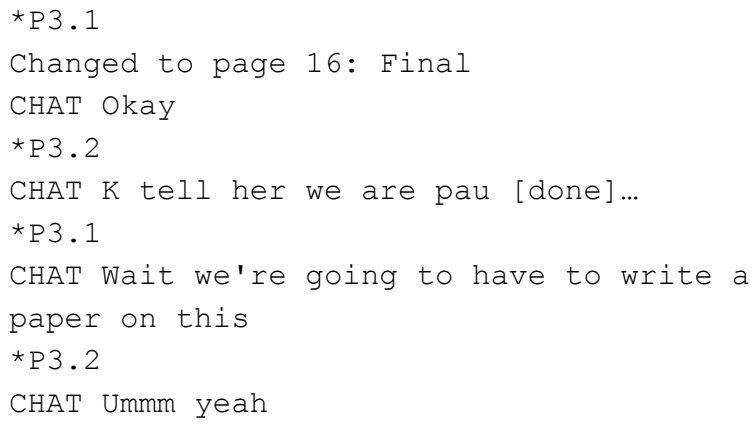




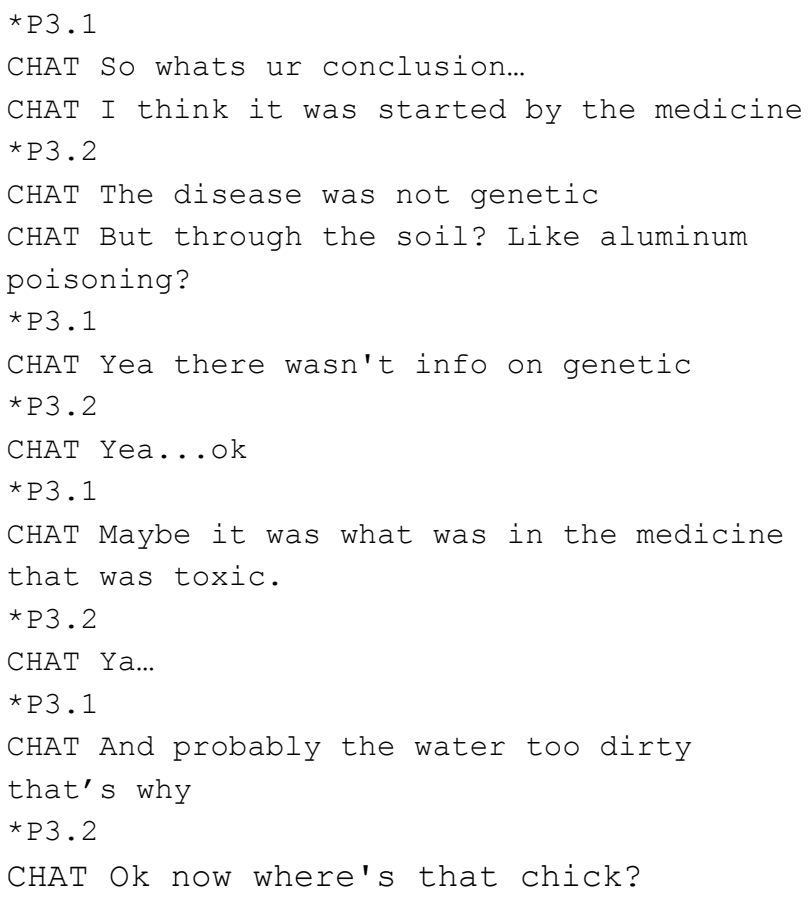

\section{Discussion}

Recall that The "Co-Present Affordances" hypothesis (H1) predicts that representational guidance is less effective when participants are not co-present, because some key mechanisms through which representations support collaboration are less effective at a distance. Therefore, we might expect that the graph's prompting for certain categories would be less influential in Distal than in the Proximal condition. The "Appropriation for Communication" hypothesis (H2) predicts that more of the communicative activity will take place in the knowledge representation medium as it is appropriated to make up for the loss of spoken "bandwidth." Therefore we would expect to see either more communications using categories provided by the representations overall, or a shift of these categories into the representational medium at the expense the verbal medium in Distal.

The quantitative results concerning categories of talk largely support the second hypothesis. To begin, a shift in emphasis on verbal versus representational activity is seen across Proximal and Distal, respectively. Participants in the Distal study represented the epistemic classification and evidential relation categories over two times more than Proximal participants. However, Proximal participants discussed epistemic classification nearly five times more than Distal participants, and evidential relation categories over nine times more than Distal participants. Clearly, Distal participants are relying on the graph for more of their communications. Yet we need to ask whether this change is universal to all communications or sensitive to the specific categories supported by the graph.

A greater percentage of acts in the Distal condition were concerned with categories provided by the graph representation (epistemic classification and evidential relations). In the case of evidential relations, the counts were similar, so this reflects a difference in the denominator: perhaps the same amount of evidential thinking is taking place in the context of less overall talk. However, a greater number of the Distal evidential relation acts are classified as "introduced," that is, as a reintroduction of the topic of evidence rather than as a continuation of an ongoing discussion of evidence. Given that many of these acts are representational, Distal participants may be using the graph medium to propose evidential relations, resulting in less overall talk but many representational introductions of evidential relations. Both numerically and in percentage, there were many more epistemic classifications in Distal. This result also supports $\mathrm{H} 2$, and is consistent with our qualitative interpretation that new objects are being created in the graph representation to propose ideas (each object creation event is also coded as a classification event).

The claim that the specific affordances of the graph influence which relations are discussed in Distal is further supported by a converse result. The Proximal condition discussed more domain talk; indeed, domain talk made up nearly five times more of the total utterances than did Distal domain talk. The graph provides no support for domain attributes and relations, so the extra work required to type concepts and propositions of an unfamiliar domain in chat would have impacted these communications more than those concerned with categories that are supported by the graph. This observation is also consistent with $\mathrm{H} 1$ : without co-presence, participants cannot as easily refer to previously represented domain concepts through gesturing.

The hypothesis statement category, which is coded when participants proposed hypotheses without classifying them as such, poses a potential challenge to H2's claim that Distal communications increase specifically in categories supported by the graph. Hypothesis statement, although not specifically supported by the graph, occurred twice as often in Distal discourse. However, most of the Distal segments in this category occur after the last page, when participants are asked to come to a final conclusion. Perhaps Distal participants are compensating for their lack of domain talk by making domain hypotheses more explicit at this stage. As predicted by $\mathrm{H} 1$ and seen in our transcript examples, activity is less tightly coordinated in Distal, so we would expect a lack of implicit agreement on the hypotheses at the end of the problem solving session. 


\section{Conclusions}

We began this study hoping to learn more about the differences between the roles of representations in Proximal and Distal synchronous collaboration. Two competing hypotheses were advanced without prejudice:

(H1) that visual knowledge representations would play less of a role in guiding discourse because without co-presence they do not as easily function to convey "taken as shared" information or to support gestural deixis; or

(H2) that visual knowledge representations would play a greater role in supporting discourse because participants would make use of them to make up for the reduced bandwidth of the verbal modes of interaction.

Our quantitative results provide adequate evidence for the second hypothesis. Talk in categories supported by the knowledge representation software (evidential relation and epistemic classification) increased online, and there was a clear shift to a greater number of communicative acts being undertaken in the representational medium.

This is not to say that we have rejected $\mathrm{H} 1$. Our informal review of the transcripts shows many examples of poorly coordinated activity in the online groups, such as disconnects between the activity in the workspace and the verbal activity in the chat. $\mathrm{H} 1$ and $\mathrm{H} 2$ are not in direct conflict, and may be synthesized as follows: Lack of mutual awareness of orientation towards shared representations may result in poorer coordination of immediate activity and the thinking behind it ( $\mathrm{H} 1)$. At the same time, greater reliance may be placed on those very representations as the medium through which activity takes place, biasing activity towards actions best supported by the representations (H2).

With respect to the design of software for on-line learning, this study suggests that close attention must be paid to the design of all representations as affordances for discourse and media that might be appropriated as a channel through which discourse takes place, regardless of whether the representation was originally intended to be a discourse medium. This conclusion also highlights the necessity of coordinating multiple representations. If users are able to modify more than one type of representation, the discourse process will not be confined to the medium provided for discourse: it will be distributed across all mutable representations. Therefore the software should support fluid cross talk between all representations by making the relationships between different representations and between acts on those representations clear $[1,6,13,18]$.

Our future work will focus primarily on asynchronous rather than synchronous online collaboration. This study has helped form a bridge to that work. Yet there remains much to be studied in the proximal versus distal paradigm. The roles of representations can be studied with different tasks, populations, and types of representations. Studies undertaken in classroom or other learning environments would offer greater external validity than this laboratory study. Data analysis might profitably focus on qualitative aspects of the roles that representations play in learners' interactions, using methodologies such as interaction analysis [8]. Whether undertaken in face-to-face, synchronous distal, or asynchronous conditions, we believe that further studies of representational affordances for collaborative interaction will be fruitful for development of both theory and practice.

\section{Acknowledgement}

We are grateful to Bin Ma and Hongli Xiang for their development of the software used in this research. This work was supported by the National Science Foundation under Grant No. 9873516. Any opinions, findings, and conclusions or recommendations expressed in this paper are those of the authors and do not necessarily reflect the views of the National Science Foundation.

\section{References}

[1] Ainsworth, S. E., Bibby, P. A., \& Wood, D. J. (1998). Analysing the Costs and Benefits of Multi-Representational Learning Environments. In M. W. van Someren, P. Reimann, H. P. A. Boshuizen, \& T. de Jong (eds.), Learning with Multiple Representations, Amsterdam: Elsevier Science, Ltd, 1998, pp. 120-134.

[2] Burgoon, J. K., Bonito, J., Ramirez, A., Kam, K., Dunbar, N., \& Fischer, J. (2002). Testing the interactivity principle: Effects of mediation, propinquity, and verbal and nonverbal modalities in interpersonal interaction. Journal of Communication 52: 657-677.

[3] Clark, H.H. \& Brennan, S.E. (1991). Grounding in Communication. In L.B. Resnick, J.M. Levine and S.D. Teasley (eds.), Perspectives on Socially Shared Cognition, American Psychological Association, pp. 127-149.

[4] Doerry, E. (1996). An Empirical Comparison of Copresent and Technologically-Mediated Interaction based on Communicative Breakdown. Ph.D. Dissertation, Department of Information and Computer Science, University of Oregon. CISTR-96-01.

[5] Herring, S.C. (1999, January). Interactive coherence in CMC. In Proceedings of the $32^{\text {nd }}$ Hawai'i International Conference on the System Sciences (HICSS 32). (CD-ROM). Maui, Hawai'i: Institute of Electrical and Electronics Engineers, Inc. (IEEE). 
Proceedings of the 36th Hawai'i International Conference on the System Sciences (HICSS-36), January 6-9, 2001, Hilton Waikoloa Village, Hawai i (CD-ROM), Institute of Electrical and Electronics Engineers, Inc. (IEEE).

[6] Hoadley, C. \& Enyedy, N. (1999). Between Information and Communication: Middle Spaces in Computer Media for Learning. In Proceedings of the Computer Support for Collaborative Learning (CSCL) 1999 Conference, C. Hoadley \& J. Roschelle (Eds.) Dec. 12-15, Stanford University, Palo Alto, California. Mahwah, NJ: Lawrence Erlbaum Associates, pp. 242-251.

[7] Hundhausen, C. D., \& Douglas, S. A. (In press). Low fidelity algorithm visualization. Journal of Visual Languages and Computing.

[8] Jordan, B. \& Henderson, A. (1995). Interaction analysis: Foundations and practice. Journal of the Learning Sciences, 4(1), 39-103.

[9] Olson, G. M. \& Olson, J. S. (1997). Research on computersupported cooperative work. In M. Helander, T. K. Landauer, \& P. Prabhu (Eds), Handbook of Human-Computer Interaction (2nd Edition), Amsterdam: Elsevier.

[10] Russel, T. L. (1999). The No Significant Difference Phenomenon. Chapel Hill, NC: Office of Instructional Telecommunications, North Carolina University. See also http://teleeducation.nb.ca/nosignificantdifference/ (accessed August 24, 2002).

[11] Schank, P., \& Kozma, R. (in press). Learning Chemistry Through the Use of a Representation-Based Knowledge Building Environment. Journal of Educational Multimedia and Hypermedia.

[12] Suthers, D. (2001a). Towards a Systematic Study of Representational Guidance for Collaborative Learning Discourse. Journal of Universal Computer Science 7(3), 2001. Online:

http://www.jucs.org/jucs_7_3/towards_a_systematic_study

[13] Suthers, D. (2001b). Collaborative Representations: Supporting Face to Face and Online Knowledge-building Discourse. Proceedings of the 34th Hawai $i$ International Conference on the System Sciences (HICSS-34), January 3-6, 2001, Maui, Hawai i, (CD-ROM), Institute of Electrical and Electronics Engineers, Inc. (IEEE).
[14] Suthers, D. \& Hundhausen, C. (2001). Learning by Constructing Collaborative Representations: An Empirical Comparison of Three Alternatives. In P. Dillenbourg, A. Eurelings, K. Hakkarainen (Eds.) European Perspectives on Computer-Supported Collaborative Learning, Proceedings of the First European Conference on Computer-Supported Collaborative Learning, Universiteit Maastricht, Maastrict, the Netherlands, March 22-24 2001, pp. 577-584.

[15] Suthers, D. \& Hundhausen, C. (2002). The Effects of Representation on Students' Elaborations in Collaborative Inquiry, Proceedings of the Computer Support for Collaborative Learning (CSCL) 2002 Conference, Boulder, Colorado, January 7-11, 2002, pp.472-480

[16] Suthers, D, Connelly, J., Lesgold, A., Paolucci, M., Toth, E., Toth, J., and Weiner, A. (2001). Representational and Advisory Guidance for Students Learning Scientific Inquiry. In K. D. Forbus and P. J. Feltovich (Eds.) Smart Machines in Education: The Coming Revolution in Educational Technology. Menlo Park: AAAI Press, 2001, pp. 7-35.

[17] Toth, E., Suthers, D. \& Lesgold, A. (2002). Mapping to know: The effects of representational guidance and reflective assessment on scientific inquiry skills. Science Education 86(2): 264-286.

[18] Turoff, M., Hiltz, S. R., Bieber, M., Fjermestad, J., \& Rana, A. (1999). Collaborative discourse structures in computer mediated group communications. Journal of Computer Mediated Communication 4(4). Online: http://jcmc.huji.ac.il/

[19] Walther, J. B. (1994). Anticipated ongoing interaction versus channel effects on relational communication in computermediated interaction. Human Communication Research 20(4), 473-501. 